\title{
Advances in Healthcare Technology: Shaping the Future of Medical Care
}

\author{
G. Spekowius and T. Wendler, eds. \\ Dordrecht, The Netherlands: Springer, 2006, 538 pages, $\$ 159$
}

In the mid 1960s Gordon Moore, cofounder of Intel Corp., professed that the number of transistors on an integrated circuit doubles every $18 \mathrm{mo}$. Later, this observation became "Moore's Law," expressing a doubling of computer power every 18 mo. For electronic equipment, especially medical imaging systems, this doubling of processing power also implies greater availability of network bandwidth, larger storage capacity, smarter computer algorithms, and better understanding of clinical information. This new technology entails improvement in disease prevention, early diagnosis, and reduction of patient discomfort. Advances in Healthcare Technology: Shaping the Future of Medical Care focuses on the impact of Moore's Law on medical imaging by discussing new medical technologies, as well as changes technology has made and will continue to make in socioeconomics and healthcare politics.

Keeping abreast of new technologic breakthroughs is a challenging task, with hundreds of new articles being published each month in scientific and medical journals. Never have so many people been involved in research as today. As the editors state, an overview of recent medical research and development with topics addressing the future of medical care is needed to help us understand the complex process of healthcare technology.

Advances in Healthcare Technology is the sixth volume of the Philips Research Book Series focusing on the latest technology in the medical innovation process linked to both industry and academic research centers. The book consists of 31 brief chapters divided into 6 sections written by expert authors from their respected scientific fields. Each chapter is supported by figures, tables, and photographs. The sections are independent of one another and cover healthcare trends, diagnostic imaging, combinations of diagnostic imaging with therapy, molecular medicine, medical informatics, and personal healthcare devices. Within this vast array of information is a mix of state-of-the-art overviews, projections on the future, and discussions of healthcare trends relevant to researchers, hospitals, and individual patients.

Some noteworthy chapters include image-guided therapy for the interventional radiologist and surgeon using multimodality hybrid imaging technologies. Such devices that are discussed include CT-integrated robotic systems for percu- taneous needle insertion, electromagnetic tracking for laparoscopic positional correction, and fusion of live ultrasound imaging with CT datasets. The goal of these devices would allow image-based treatment planning, guidance, monitoring, and controlling of treatment effectiveness. As stated by one of the authors, the ultimate goal of having a "tricorder" device, as used by Dr. McCoy on Star Trek, which could hover over an afflicted area and both sense and cure the disease may not be light years away.

Other interesting chapters discuss technologic imaging innovations such as 3-dimensional rotational x-ray imaging, coronary MR angiography, and 4-dimensional ultrasound. In the section on molecular imaging is a fascinating chapter about using targeted nanoparticles for noninvasive phenotypic characterization of pathologic conditions, with an end of providing individual custom-tailored therapies. The chapters on personal healthcare discuss future scenarios in which individuals take increasing responsibility for managing their own healthcare, especially through their use of electronic remote monitoring devices and day-to-day interaction via a variety of platforms such as Web browsers, cell phones, and digital medium devices.

This book has some limitations that require mentioning. Several chapters cover "basic mathematics" that in reality are quite complex and more appropriate for a research article than for a book intended for a wider audience. Other chapters fail to describe current advances in medical technology and consist mainly of ill-summarized histories of established technologies. Being based principally on Philips research programs surprisingly is not a key limitation of the book.

In summary, Advances in Healthcare Technology is an interesting collection of emerging technologies in the field of medical research. The book should be of value to both researchers and physicians interested in the development and future of modern medicine, in which new technology plays a predominant role. 